\title{
Size- and Temperature-Dependent Intraband Optical Properties of Heavily n-Doped PbS Colloidal Quantum Dot Solid-State Films
}

Iñigo Ramiro,* Biswajit Kundu, Mariona Dalmases, Onur Özdemir, María Pedrosa, and Gerasimos Konstantatos*

Cite This: ACS Nano 2020, 14, 7161-7169

Read Online

\section{ACCESS \\ Llll Metrics \& More \\ Article Recommendations \\ Supporting Information}

ABSTRACT: Steady-state access to intraband transitions in colloidal quantum dots (CQDs), via doping, permits exploitation of the electromagnetic spectrum at energies below the band gap. CQD intraband optoelectronics allows envisaging cheap mid- and long-wavelength infrared photodetectors and light-emitting devices, which today employ epitaxial materials. As intraband devices start to emerge, thorough studies of the basic properties of intraband transitions in different CQD materials are needed to guide technological research. In this work, we investigate the size and temperature dependence of the intraband transition in heavily $\mathrm{n}$-doped $\mathrm{PbS}$ quantum dot (QD) films. In the studied QD size range $(5-8 \mathrm{~nm})$, the intraband energy spans from 209 to $151 \mathrm{meV}$. We measure the intraband absorption coefficient of heavily doped $\mathrm{PbS} \mathrm{QD}$ films to be around $2 \times 10^{4} \mathrm{~cm}^{-1}$, proving that intraband absorption is as strong as interband absorption. We demonstrate a negative dependence of the intraband energy with temperature, in contrast to the positive dependence of the interband transition. Also opposite to the interband case, the temperature dependence of the intraband energy increases with decreasing size, going from $-29 \mu \mathrm{eV} / \mathrm{K}$ to $-49 \mu \mathrm{eV} / \mathrm{K}$ in the studied size range.

KEYWORDS: quantum dots, intraband, lead sulfide, absorption coefficient, temperature dependence

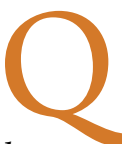

uantum dots (QDs) are zero-dimensional solids that exhibit atomic-like electronic structures, with discrete electronic levels separated by a true zero density of states. The formidable control in size and composition of colloidal quantum dots (CQDs) has enabled experimental study of fundamental quantum physics as well the utilization of these materials for a wide variety of applications in the fields of light emission ${ }^{1}$ and detection, ${ }^{2}$ photovoltaics, ${ }^{3}$ and field effect transistors. ${ }^{4}$ For semiconductor (SC) device engineering, one key advantage that CQDs offer compared to conventional bulk materials is band gap tunability. The band gap of CQDs can be increased from the value of their respective bulk counterpart in a controlled manner, by decreasing the size of the dots. This characteristic allows QDs of large band gap bulk SCs, such as cadmium chalcogenides, to be tuned in the entire range of visible light, ${ }^{5}$ QDs of low band gap SCs, such as lead chalcogenides, to span over the near and short-wave infrared (NIR and SWIR), ${ }^{6}$ and QDs of semimetals, such as mercury chalcogenides, to span over the mid- and long-wave infrared (MWIR and LWIR). ${ }^{7}$

Over the last two decades, yet another degree of freedom in the band gap tunability of CQDs has been explored. By highly populating, via doping, either the conduction band $(\mathrm{CB})$ with electrons or the valence band (VB) with holes, it is possible to access optically intraband transitions in those bands, as demonstrated for cadmium ${ }^{8,9}$ and lead chalcogenides. ${ }^{10}$ The emergence of intraband absorption is coupled to progressive bleaching of interband absorption, as the $\mathrm{CB}$ or VB becomes more populated. Intraband transitions, taking place between electronic states within a band, are less energetic than the

Received: March 8, 2020

Accepted: May 12, 2020

Published: May 12, 2020

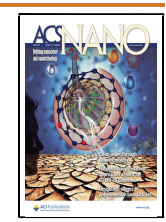


conventionally exploited interband transitions and can even be smaller than the band gap of the bulk counterpart, thus allowing exploitation of part of the electromagnetic spectrum forbidden for a given bulk SC material. To give an example of its potential, fine-tuning of and access to intraband transitions in CQDs could lead to photodetectors and light sources in the MWIR and LWIR ${ }^{11}$ made of relatively high bandgap, cheap, and nontoxic materials, replacing the current technology based on epitaxially grown $\mathrm{HgCdTe}$, InGaAs and $\mathrm{GaInSb}^{12}$ Consequently, work in this field is becoming more and more intense, and steady-state probing of intraband transitions has already been achieved in several materials. ${ }^{13}$ However, until recently only mercury chalcogenide CQDs have exhibited stable high doping that allowed fabrication of intraband photodetectors. ${ }^{1114,15}$ Recently, robust heavy doping has been demonstrated in $\mathrm{PbS}$ CQD films, ${ }^{16}$ enabling intraband absorption and photodetection in the MWIR and LWIR, ${ }^{17}$ at energies smaller than the $\mathrm{PbS}$ bulk band gap. At this point, studies of the basic properties of intraband transitions in CQDs are needed to guide the development of intrabandbased devices and applications. In this work, we investigate the size and temperature dependence of intraband transitions in the $\mathrm{CB}$ of $\mathrm{PbS}$ CQDs. We report the intraband absorption coefficient of $\mathrm{PbS}$ QD films and discuss the size dependence of the oscillator strength of the first intraband transition in $\mathrm{PbS}$ QDs.

\section{RESULTS AND DISCUSSION}

PbS CQDs and Doped Films Used for the Study. Figure 1 shows absorbance spectra of the PbS CQDs used in

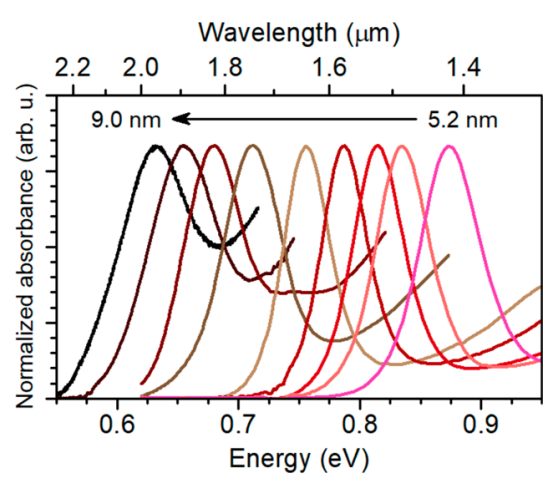

Figure 1. Absorbance spectra, normalized to the maximum of the exciton peaks, of oleic-acid capped $\mathrm{PbS}$ CQDs dispersed in toluene. The QD diameter of the different solutions ranges between 5.2 and $9.0 \mathrm{~nm}$.

this study, which evidence good control on the QD size (diameter) in the 5-9 $\mathrm{nm}$ range and low size dispersion. QD size has been obtained using the empirical model for oleic-acid capped QDs reported in ref 6 after fitting the exciton energy in the absorbance spectra.

After synthesis, we fabricated doped films following the method reported in ref 16 . The films are spin-coated layer-bylayer on silicon substrates, exchanging the original oleic acid ligand with iodine. We use semi-insulating (lightly doped) silicon substrates throughout the whole study because the different measurements performed require transparency in the $1-12 \mu \mathrm{m}$ range. After formation, the film is infiltrated and capped with alumina by atomic layer deposition, a method that has been proven to provide n-doping in $\mathrm{PbSe}^{18}$ and $\mathrm{ZnO}^{19,20}$
QDs. The resulting PbS CQD films are heavily n-doped ( $\left.10^{19} \mathrm{~cm}^{-3}\right)^{16}$ and, consequently, exhibit steady-state intraband absorption in the $\mathrm{CB} .{ }^{17}$ Figure $2 \mathrm{~b}$ shows the first two interband

(a)

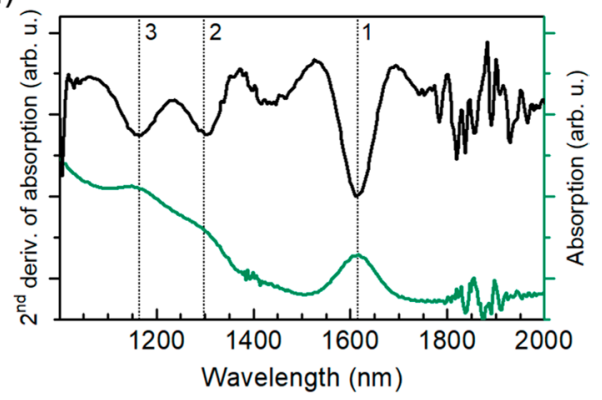

(b)

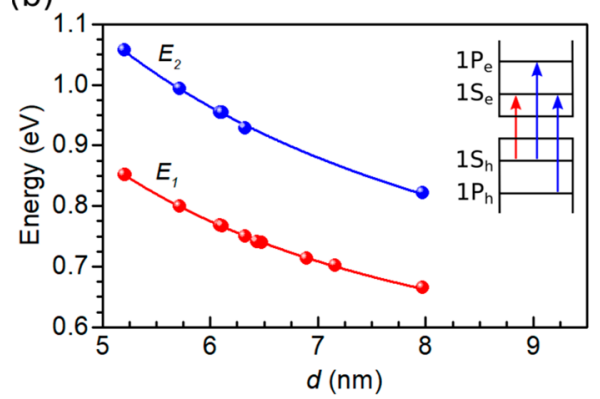

Figure 2. Interband absorption in heavily n-doped PbS CQD films. (a) Second-derivative analysis of the absorption spectrum. Vertical lines indicate the first three excitons. (b) Energy of the first two excitons, corresponding to lines 1 and 2 in (a) as a function of $d$. The lines are fits, as described in the main text. The inset illustrates the transitions of the first (red arrow) and second (blue arrows) excitons. Resulting from the n-doped character of the QDs, the measured $E_{2}$ originates exclusively from $1 S_{h}-1 P_{e}$ transitions and not from $1 \mathrm{P}_{\mathrm{h}}-1 \mathrm{~S}_{\mathrm{e}}$ transitions.

excitons in the doped films as a function of the dot diameter, $d$, obtained via analysis of the absorption spectra, as shown in Figure 2a. Note that for some samples, $E_{2}$ could not be accurately fitted, hence the reduced number of data points for $E_{2}$ in Figure $2 \mathrm{~b}$. We found that the first exciton is red-shifted in the doped films as compared to the as-synthesized solutions (Supporting Figure S1). We used this exciton-shift fingerprint as well as the doping level of the samples (discussed later and shown in Figure 4d) to discard oxidation of the dots before or during film formation, thus guaranteeing proper sample quality for further quantitative studies. We found that the samples that showed signs of oxidation (slight blue-shift of the first exciton) also exhibited lower doping levels.

We have fitted the first exciton, $E_{1}\left(1 S_{\mathrm{h}}-1 S_{\mathrm{e}}\right)$, and the second exciton, $E_{2}\left(1 S_{\mathrm{h}}-1 \mathrm{P}_{\mathrm{e}}\right)$, with eqs 1 and 2 :

$$
E_{1}(\mathrm{eV})=0.41+\frac{1.50}{d}+\frac{4.2}{d^{2}}
$$

with a fit correlation coefficient, $R^{2}>0.99$.

$$
E_{2}(\mathrm{eV})=0.41+\frac{3.09}{d}+\frac{1.4}{d^{2}}
$$

with $R^{2}>0.99$.

The constant term $0.41 \mathrm{eV}$ is the bulk band gap of $\mathrm{PbS}, E_{\mathrm{g}}$, at room temperature (RT). The size-dependent $1 / d^{2}$ represents the confinement energy characteristic of particles 
in a well under the effective mass approximation. The $1 / d$ term is an empirical observation required to fit $E_{1}$ and $E_{2}$, as it was the case in previous studies, ${ }^{6,21}$ indicating that corrections to this approximation are needed. In both transitions, the $1 / d$ term is dominant over the quantum confinement one, as it is the case in as-synthesized PbS CQDs. ${ }^{6}$ In fact, for $E_{2}$, data can be fitted without the $1 / d^{2}$ term, in the $5 \mathrm{~nm}<d<8 \mathrm{~nm}$ range, with $R^{2}>0.99$. However, the fact that the $1 / d^{2}$ term of $E_{2}$ is almost negligible in this size range is not particular of doped dots. Cademartiri et al. ${ }^{21}$ showed that the second exciton in undoped $\mathrm{PbS}$ CQDs dispersed in solution deviates from the expected behavior for $d>5 \mathrm{~nm}$, which caused $E_{2}$ to not be fitted to an equation of the form of eq 1 in the $4-7 \mathrm{~nm}$ size range. Therefore, although we expect eq 1 to be valid for $d<5$ $\mathrm{nm},{ }^{6,21}$ we do not expect the same for eq 2.

There is not a clear explanation for the peculiar size dependence of $E_{2}$. Although it was a cause of controversy in the past, there is now a consensus that it corresponds to the $1 \mathrm{~S}_{\mathrm{h}}-1 \mathrm{P}_{\mathrm{e}}$ and $1 \mathrm{P}_{\mathrm{h}}-1 \mathrm{~S}_{\mathrm{e}}$ transitions. $^{22,23}$ These two have very similar energies due to the almost identical electron and hole effective masses in $\mathrm{PbS}^{24}$ as indicated in the inset of Figure $2 \mathrm{~b}$. Both transitions should be forbidden by symmetry. However, it has been shown that parity selection rules are relaxed in $\mathrm{PbS}$ QDs, allowing optical absorption features that do not conserve parity. $^{22}$ It has been discussed that breaking of the inversion symmetry of the wave function is needed to fully explain the optical absorption spectra of $\mathrm{PbS}$ QDs. ${ }^{23}$ Ref 21 shows yet another higher-energy transition (seventh exciton) that exhibits a size dependence behavior similar to $E_{2}$, with a sudden change in trend for $d>5 \mathrm{~nm}$, and discusses the possibility that this transition originates from a separate corner of the Brillouin zone. Here, we propose another explanation for the sudden change in the size dependence of $E_{2}$. It has been reported that the morphology of $\mathrm{PbS} \mathrm{QDs}$ changes with size, evolving from an octahedral shape, for $d<3 \mathrm{~nm}$, to a cuboctahedral shape, for $d>4 \mathrm{~nm} .^{25}$ Small QDs have Pb-rich (111) facets, whereas in larger dots, S-rich (100) facets emerge. We argue that the change in morphology in large dots, having exposed sulfur atoms, may contribute to the formation of new states that modify the energy of the second (and possibly the seventh) interband transition.

Size Dependence of the Intraband Transition. We have measured the absorption spectra of the intraband transitions in our films, $A_{\text {film }}$ by means of transmission and reflection measurements via Fourier transform infrared spectroscopy. Our samples consist of a thin film $(\sim 100 \mathrm{~nm})$ of PbS QDs on top of a substrate $(\sim 300 \mu \mathrm{m})$ of semi-insulating silicon, as sketched in Supporting Figure S2. $A_{\text {film }}$ is defined as

$$
A_{\text {film }}=1-R_{\text {meas }}-T_{\text {meas }}-A_{\text {subs }}
$$

where $R_{\text {meas }}$ and $T_{\text {meas }}$ are the measured transmission and reflection, and $A_{\text {subs }}$ is the absorption of the substrate, which was previously characterized through reflection and transmission measurements (see Supporting Figure S3). Figure 3a shows that the intraband transition shifts to higher energies as the dot size decreases, owing to increased quantum confinement. Sharp features at around 0.14 and $0.15 \mathrm{eV}$ arise from the differences (such as the amount of $\mathrm{SiO}_{2}$ ) in the reference silicon substrate and the substrate of each sample (see Supporting Figure S4). Below $0.13 \mathrm{eV}$, alumina contributes to absorption (Supporting Figure S5).

The size dependence of the intraband energy, $E_{\mathrm{IB}}$, at RT is plotted in Figure $3 \mathrm{~b}$. We have fitted the results with eq 4 : (a)

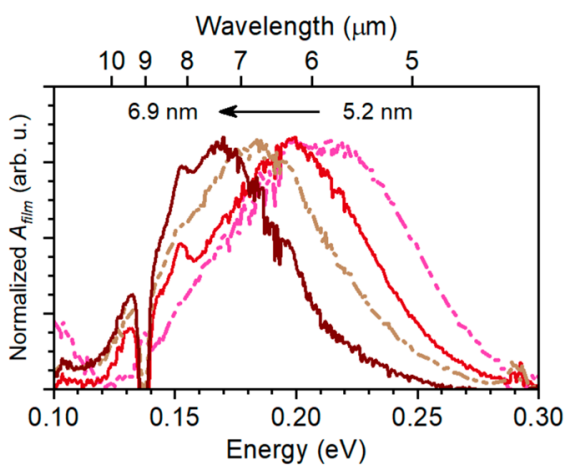

(b)

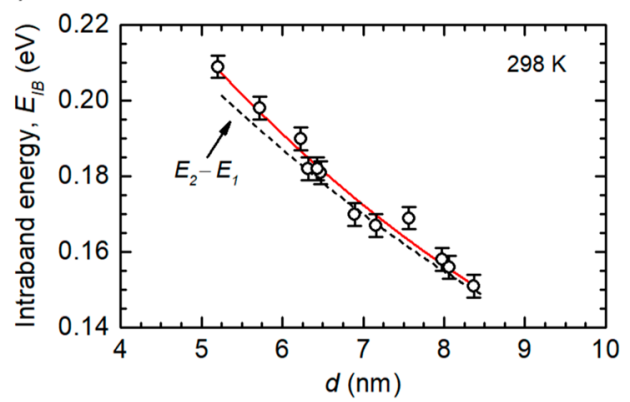

(c)

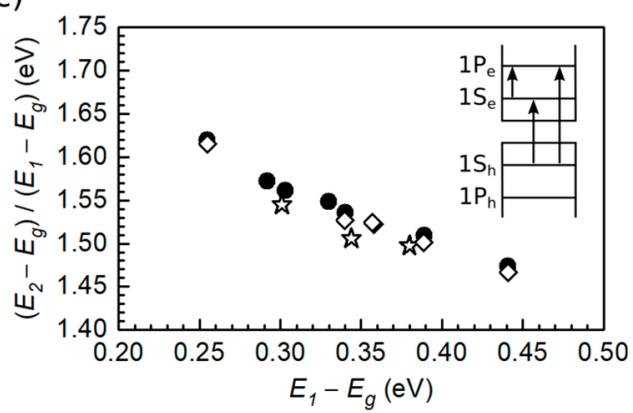

Figure 3. (a) Intraband absorption spectra of QDs of different sizes normalized to the peak maxima. (b) Energy of the intraband transition, at RT, as a function of the dot diameter. Data are obtained as the central energies of Gaussian fits of absorption spectra such as those presented in (a). The error bars are estimates of the uncertainty in the fits $( \pm 3 \mathrm{meV})$. The red solid line corresponds to eq 4 . The black dashed line represents the energy difference between the second and first excitons. (c) Second confinement energy $\left(E_{2}-E_{\mathrm{g}}\right)$ divided by the first confinement energy $\left(E_{1}-E_{\mathrm{g}}\right)$ as a function of the first confinement energy. $E_{2}$ $\left(1 S_{h}-1 P_{e}\right)$ is obtained in two ways: directly from absorption spectra at RT $(\diamond)$ and as $E_{\mathrm{IB}}\left(1 \mathrm{~S}_{\mathrm{e}}-1 \mathrm{P}_{\mathrm{e}}\right)+E_{1}\left(1 \mathrm{~S}_{\mathrm{h}}-1 \mathrm{~S}_{\mathrm{e}}\right)$ at RT $(\bullet)$ and $98 \mathrm{~K}($ 泞).

$$
E_{\mathrm{IB}}(\mathrm{eV})=\frac{1.57}{d}-\frac{2.5}{d^{2}}
$$

with $R^{2}=0.97$. The negative value of the $1 / d^{2}$ term is expected, since we expect $E_{\mathrm{IB}}$ to be equal to $E_{2}-E_{1}$ (see eqs 1 and 2). For comparison, we have plotted $E_{2}-E_{1}$ in Figure $3 \mathrm{~b}$. These results support the fact that, for all studied samples, the absorption peaks analyzed correspond to intraband transitions. As it was the case for $E_{1}$ and $E_{2}$, the $1 / d$ term represents the dominant contribution to the size dependence of $E_{\mathrm{IB}}$. This will be useful later on for the analysis of the temperature dependence of $E_{\mathrm{IB}}$. As discussed previously, eq 2 is not valid for $d<5 \mathrm{~nm}$, therefore, eq 4 should not be valid either. We do not know whether eq 2 and, hence, eq 4 still hold for $d>8.5$ 
(a)

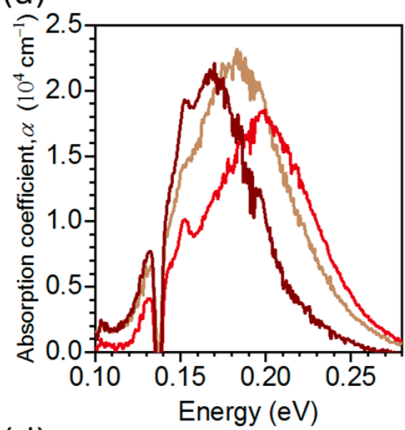

(d)

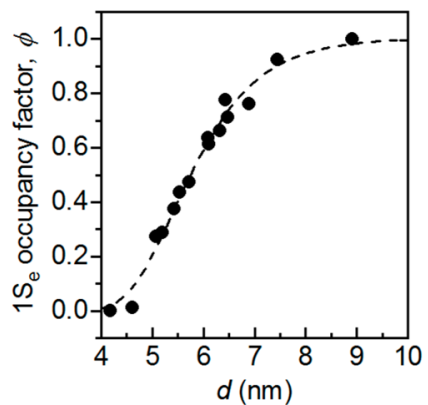

(b)

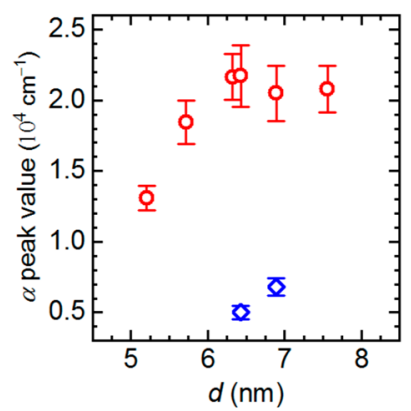

(e)

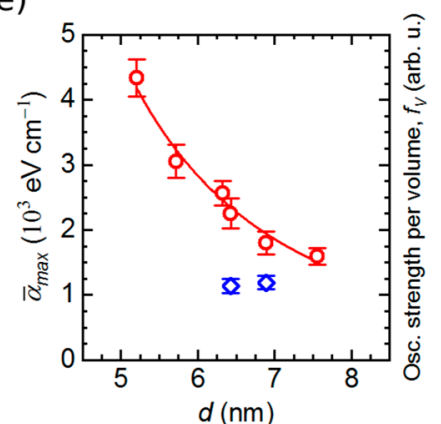

(c)

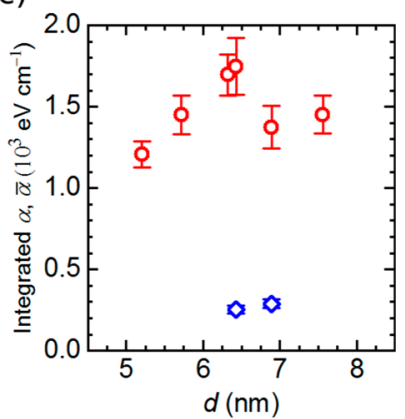

(f)

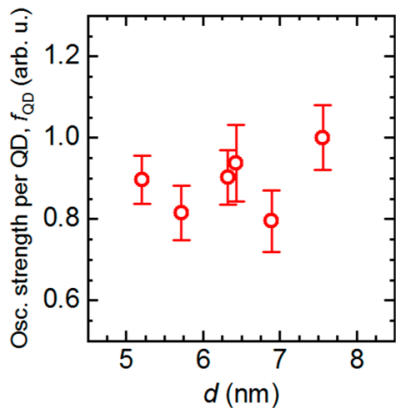

Figure 4. Absorption coefficients in heavily $\mathrm{n}$-doped PbS CQD films. (a) Absorption coefficient spectra measured in PbS QD thin films for QDs of different sizes. (b) Peak values of the intraband (red circles) and interband (blue rhombus) absorption coefficients. Values are obtained by fitting the absorption spectra to Gaussians. The error bars account for the error propagation of the uncertainty in film thickness (see Supporting Table S1) and the absorption measurements ( $5 \%$, estimated). (c) Integrated absorption coefficients. Values are obtained by integration of the fitted Gaussians. (d) Measured occupancy factor of $1 S_{\mathrm{e}}$. (e) The maximum attainable value of $\bar{\alpha}$, $\bar{\alpha}_{\max }$, and oscillator strength per volume of the intraband and interband absorption. The line is a power law fit. (f) Oscillator strength per QD of the intraband absorption.

nm. We know, however, that $E_{\mathrm{IB}}$ should get progressively closer to zero as the quantum confinement gets weaker. For dot diameters close to the exciton Bohr radius, $a_{\mathrm{B}}$, of $\mathrm{PbS}(\sim 20$ $\mathrm{nm})$, the nanocrystals are in the weak confinement regime and $E_{\mathrm{IB}}$ should approach zero.

In the framework of the effective mass approximation, the normalized confinement energy of the different interband transitions (confinement energy of exciton $i, E_{i}-E_{\mathrm{g}}$, divided by the confinement energy of the first exciton, $E_{1}-E_{\mathrm{g}}$ ) should be independent of $E_{1}-E_{\mathrm{g}}{ }^{10}{ }^{10}$ Figure $3 \mathrm{c}$ plots the normalized confinement energy of the second intraband transition. We have used two set of values for $E_{2}$, those obtained directly from absorption measurements (Figure $2 b$ ) and values obtained as $E_{1}+E_{\mathrm{IB}}$ (Figures $2 \mathrm{~b}$ and $3 \mathrm{~b}$ ). As expected, both sets of values yield very similar results. However, instead of a sizeindependent value, we observe a linear decrease of the normalized confinement energy of the second exciton for $E_{1}$ $-E_{\mathrm{g}}$ in the $0.25-0.45 \mathrm{eV}$ range $(8 \mathrm{~nm}>d>5 \mathrm{~nm})$. This behavior matches the one reported for undoped $\mathrm{PbS}$ CQDs dispersed in solution, ${ }^{21}$ where $\left(E_{2}-E_{\mathrm{g}}\right) /\left(E_{1}-E_{\mathrm{g}}\right) \approx 1.39$ for $E_{1}-E_{\mathrm{g}}>0.45 \mathrm{eV}(d<5 \mathrm{~nm})$ and increases for smaller confinement energies (larger dots). We ascribe this behavior to the fact that both $E_{1}$ and $E_{2}$ have a predominant $1 / d$ contribution over the $1 / d^{2}$ confinement energy term, as discussed in the previous section.

Figure $3 \mathrm{c}$ also shows measurements of $\left(E_{2}-E_{\mathrm{g}}\right) /\left(E_{1}-E_{\mathrm{g}}\right)$ at $98 \mathrm{~K}$. To calculate $E_{\mathrm{g}}$ at low temperatures, we used 320 $\mu \mathrm{eV} / \mathrm{K}$ as the temperature dependence of the bulk PbS band gap. ${ }^{26}$ The low-temperature values of normalized confinement energies match closely the values at RT, indicating that $E_{1}$ and $E_{2}$ at low temperatures follow similar size dependences to those shown in Figure $2 \mathrm{~b}$. The impact of temperature on the intraband transition will be studied later on.

Absorption Coefficient of Doped PbS QD Films. Characterizing the intrinsic absorption intensity of a semiconductor is crucial both for its use in practical applications and for theoretical studies. We have performed absorption measurements such as those shown in Figure $3 a$ to calculate the absorption coefficient of our films (see Methods). Figure 4a shows some of the measured absorption coefficient spectra of the intraband region. The peak values of $\alpha$ for the $1 S_{e}-1 P_{e}$ transition are plotted in Figure $4 \mathrm{~b}$ as a function of $d$. The $\alpha$ peak values and other relevant parameters of the results presented in Figure 4 are compiled in Supporting Table S1. For comparison, $\alpha$ for the interband $\left(1 S_{h}-1 S_{\mathrm{e}}\right)$ transition in the same samples is also plotted in Figure $4 \mathrm{~b}$. Note that in these absorption measurements, we have not been able to measure the interband transitions for all samples, and the reason is two-fold: For the samples with smaller QDs, the exciton energy falls out of the detector range. For the samples with larger QDs, the interband absorption peak is almost completely bleached ( $1 S_{\mathrm{e}}$ almost completely filled). The measured interband absorption coefficient in these heavily doped samples is around $0.5 \times 10^{4} \mathrm{~cm}^{-1}$. From this value, we can estimate what the interband absorption coefficient would be in undoped samples. The interband absorption peak in the doped samples has been bleached to about a quarter of its value prior to doping (Supporting Figure S6). Therefore, the interband absorption coefficient for undoped or lightly doped films would be in the order of $2 \times 10^{4} \mathrm{~cm}^{-1}$, similar to the measured value of the intraband absorption coefficient and 
close to values previously reported for undoped PbS CQDs measured in solution. ${ }^{27}$

To make a better comparison between absorption intensity in different films, Figure $4 \mathrm{c}$ shows the values of $\alpha$ integrated over the intraband (or interband) absorption range, $\bar{\alpha}$. Integration over the whole spectrum of the transition corrects for the impact on $\alpha$ peak values of differences in full width half maxima (fwhm, see Supporting Table S1) of the absorption spectra. We ascribe the differences on fwhm to different size distribution in the synthesized nanoparticles.

In our QD films, $\alpha$ and $\bar{\alpha}$ are influenced by factors extrinsic to the QDs, such as the packing density of the films (or the volume fraction occupied by the QDs) and the presence of a surrounding medium (alumina) imposed by the doping procedure, which alters the effective dielectric function of the film. ${ }^{27}$ For fundamental studies that require comparison with theory, the oscillator strength is a more adequate parameter. The oscillator strength is an intrinsic property of materials related to the probability of an electronic quantum transition upon the presence of a resonant electromagnetic field. The integrated absorption coefficient of the QD film is directly related to the oscillator strength per volume, $f_{\mathrm{V}}{ }^{28}$ The oscillator strength per particle, $f_{\mathrm{QD}}$, instead, determines the radiative lifetime. Because we ignore the dielectric constant of our material in the energy range of our study as well as the exact volume fraction occupied by the QDs in the films, we cannot know precisely the intrinsic absorption coefficient of the QD material, and consequently, we cannot make a quantitative study of the oscillator strength of our particles. However, under the assumption that the film packing density and dielectric constant are similar in all samples, we are able to evaluate in qualitative terms the dependence of $f_{\mathrm{QD}}$ with $d$. In doing so, the doping level of our samples needs to be determined. $\mathrm{PbS}$ QDs can accommodate up to 8 electrons in the $1 S_{\mathrm{e}}$ state. $^{24}$ Therefore, we define the $1 \mathrm{~S}_{\mathrm{e}}$ occupancy factor $\varnothing=\frac{n_{\mathrm{QD}}}{8}$, where $n_{\mathrm{QD}}$ is the average doping level of the QDs in the film (measured in electrons per QD, see Methods). Figure $4 \mathrm{~d}$ shows the measured $\varnothing$ as a function of $d$. It shows a higher occupancy in larger dots. Note that the phenomenological observation of increased doping in larger sized QDs has been previously reported in other CQD materials, such as $\mathrm{CdSe}^{29}$ and HgSe. ${ }^{14}$ In the doping method employed in this work, the increasing doping with size seems to be strongly related to the size-dependent presence of exposed sulfur sites to undergo halide substitution, ${ }^{16}$ which in turn leads the Fermi level to approach and cross the $1 S_{\mathrm{e}}$ state. Whereas the oscillator strength defines the probability of a quantum absorption process to take place, the occupancy of the initial and final states has to be additionally considered to calculate the absorption coefficient. ${ }^{30}$ Thus, looking at the intraband transition, in undoped $\mathrm{PbS}$ QDs (empty $1 \mathrm{~S}_{\mathrm{e}}$ ) $\bar{\alpha}=0$, because the initial state is empty, whereas $f_{\mathrm{QD}} \neq 0$, because it is independent of the occupancy of the initial and final states. Therefore, for a proper comparison of the oscillator strength in different samples, $\bar{\alpha}$ has to be corrected by $\emptyset$. Let us call $\bar{\alpha}_{\max }$ the maximum attainable value of $\bar{\alpha}$. For intraband transitions, $\bar{\alpha}$ will be maximum at $\varnothing=1$, whereas for interband transitions, $\bar{\alpha}$ will be maximum at $\varnothing=0$. Hence, we define $\bar{\alpha}_{\max }=\bar{\alpha} / \varnothing$ for intraband transitions and $\bar{\alpha}_{\max }=\bar{\alpha} /(1-\varnothing)$ for interband transitions. This correction allows us to relate directly the measured $\bar{\alpha}$ to $f_{\mathrm{V}}$, as shown in Figure 4e. Note that, implicitly, we are assuming that, resulting from Fermi-Dirac statistics, the $1 \mathrm{P}_{\mathrm{e}}$ state is mostly empty at RT for all samples, given $E_{\mathrm{IB}}>$ $0.15 \mathrm{eV}$ (Supporting Figure S7). The results show that the oscillator strength of the intraband transition is in the same order and even higher than that of the interband transition. For the intraband case, we have fitted $f_{\mathrm{V}}$ to a power law equation, resulting in an exponent value of -2.7 and $R^{2}=0.98 . f_{\mathrm{QD}}$ is obtained as $f_{\mathrm{QD}}=f_{\mathrm{V}} \times V$, where $V$ is the volume of a QD of diameter $d$. Figure $4 \mathrm{f}$ plots the size dependence of $f_{\mathrm{QD}}$, assuming the QDs to be spherical.

The absorption strength of interband transitions has been studied for different semiconductor nanocrystals by measuring the absorption coefficient, the molar extinction coefficient, or the absorption cross section. ${ }^{6,21,31-37}$ Early calculations of the strength of interband transitions suggested that $f_{\mathrm{QD}}$ for $E_{1}$ should be independent of the dot size, so long as the dots are in the strong confinement regime. ${ }^{28}$ More recently, it was pointed out that further theoretical studies, going beyond the effective mass approximation, are needed to explain the experimental results obtained for different materials. ${ }^{27}$ Fewer studies are reported on interband transitions. Ref 14 calculates a linear dependence with size of the oscillator strength of the interband transition in $\mathrm{HgSe}$ QDs. Some theoretical works have calculated the oscillator strength of intraband transitions for QDs of different materials. ${ }^{30,38,39}$ However, because of the particularities of each study, none of these works can be directly related to our case. Our results indicate that the oscillator strength per particle of the intraband transition in $\mathrm{PbS}$ QDs has a weak dependence on $d$ in the studied size range. We hope that this work will motivate theoretical studies of the oscillator strength of intraband transitions in lead chalcogenide QD materials.

Temperature Dependence of the Intraband Transition. Analysis of the temperature dependence of the interband and intraband transitions is also fundamental for the development of theoretical models that can predict the optical properties of CQD materials as well as in their applications to light emitters and photodetectors. The temperature dependence of the interband transition in cadmium and lead chalcogenide CQDs has been extensively analyzed. ${ }^{37,40-45}$ In the case of $\mathrm{PbS}$, Olkhovets et al. ${ }^{40}$ showed that $E_{1}$ is nearly independent of temperature in small dots $(d<$ $4 \mathrm{~nm}$ ), as expected for atomic-like energy levels. As the dots get larger, $E_{1}$ has an increasing positive dependence with temperature, approaching the temperature dependence of bulk $\mathrm{PbS}$ as quantum confinement is reduced. Different theoretical models have been used to explain the experimental results, taking into account the impact of lattice expansion, ${ }^{40}$ electron-phonon (el-ph) interactions, ${ }^{40,41,43}$ and effective mass. ${ }^{44}$ From these works, it is concluded that el-ph interactions play a predominant role in the temperature dependence of $E_{1}$.

We have measured the intraband absorption in our heavily doped samples in the temperature range of 98-298 K (see Methods). Figure 5a,b shows measurements for two samples with different dot sizes. The first noticeable feature is the redshift of $E_{\mathrm{IB}}$ as temperature increases, conversely to the reported blue-shift of $E_{1}$ in undoped PbS CQDs. ${ }^{40}$ A similar effect was reported for HgSe QDs. ${ }^{14}$ The positive dependence of $E_{1}$ with temperature is preserved in doped QDs, as shown in Figure 5c. Another interesting aspect is the apparent increase in intraband absorption intensity upon sample cooling. We think that this is an intrinsic property of the material, not due to a variation of $\varnothing$ with temperature, since the doping level of the dots has been 
(a)

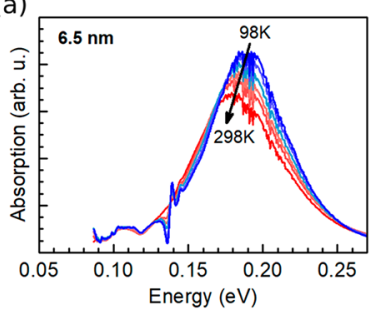

(c)

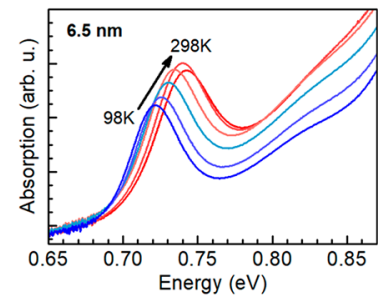

(b)

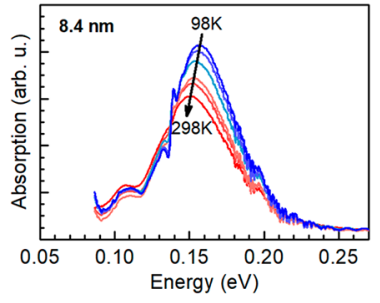

(d)

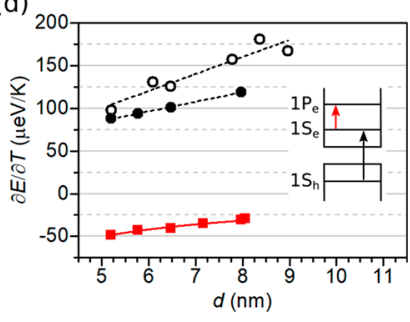

dependence of interband transitions in $\mathrm{PbS} \mathrm{QDs}$ is smaller for higher energies. ${ }^{40}$ Thus, the temperature dependence of $E_{2}$ is expected to be weaker than that of $E_{1}$. Since $E_{\mathrm{IB}}=E_{2}-E_{1}$, it follows that $\partial E_{\mathrm{IB}} / \partial T<0$. In addition, since the energy difference between $E_{2}$ and $E_{1}$ is larger for smaller dots, $\partial E_{\mathrm{IB}} / \partial T$ should increase (in magnitude) as the dot size is reduced.

In our lightly doped films, the values of $\partial E_{1} / \partial T$ are similar to those previously reported for undoped QDs. ${ }^{40}$ Upon heavy doping, $\partial E_{1} / \partial T$ is reduced. The difference in $\partial E_{1} / \partial T$ between the heavily doped and lightly doped samples increases with dot size and, hence, with $\varnothing$ (see Figure 4). For samples with $d=5$ $\mathrm{nm}(\varnothing \sim 0.1)$, both values are very close. We deduce that upon occupation of $1 S_{\mathrm{e}}$, the el-ph interaction is modified, resulting in a reduced contribution to the temperature dependence of $E_{1}$.

Next, we analyze the size dependence of $\partial E_{\mathrm{IB}} / \partial T$ on the basis of empirical results discussed above. We know, through eqs 1 and 2, that at RT both $E_{1}$ and $E_{2}$ are dominated by the 1 / $d$ term. In addition, Figure 3 indicates that this behavior is maintained at low temperatures. It follows that $\partial E_{\mathrm{IB}} / \partial T$ will have a $1 / d$ dependence with size. Figure 5 shows a fit $\left(R^{2}=\right.$ 0.96 ) of the measured data to $C / d$, where $C$ is a constant. For large values of $d, \partial E_{\mathrm{IB}} / \partial T$ tends to zero, since $E_{\mathrm{IB}} \rightarrow 0$ because of the relaxation of the quantum confinement as $d \rightarrow a_{B}$.

\section{CONCLUSIONS}

We have performed size- and temperature-dependent optical studies of the intraband transition in densely packed films of $\mathrm{PbS}$ QDs, thus providing valuable data that can be directly used in device design and engineering. The absorption coefficient of the intraband transition is in the order of the absorption coefficient of the interband transition $\left(10^{4} \mathrm{~cm}^{-1}\right)$. This result implies that thin-film PbS intraband optoelectronics can be envisaged. Additionally, dots with semioccupied $1 S_{e}$ (only partially bleached interband) exhibit strong absorption in both interband and intraband transitions. These dual-band strong absorbers may be interesting for multigap optoelectronic devices such as intermediate band solar cells, ${ }^{46}$ which currently experience a major setback related to weak absorption. $^{47}$

Our measurements reveal absorption in the first intraband transition $\left(1 \mathrm{~S}_{\mathrm{e}}-1 \mathrm{P}_{\mathrm{e}}\right)$. However, absorption at more energetic intraband transitions (from $1 S_{e}$ to higher levels such as $1 D_{e}$, $2 \mathrm{~S}_{\mathrm{e}}$, and $\left.1 \mathrm{~F}_{\mathrm{e}}\right)^{22}$ could not be detected (Supporting Figure S10), likely because these transitions are either forbidden or much weaker than the first intraband transition in the studied size range. Our results further indicate that the oscillator strength per particle of the intraband transition has a weak dependence on the dot size.

\section{METHODS}

PbS Colloidal QD Synthesis. PbS QDs synthesis was adapted from a previously reported multi-injection procedure. ${ }^{48}$ Briefly, a mixture of lead oxide ( $\mathrm{PbO}$ ), 1-octadecene (ODE), and oleic acid was degassed overnight at $90{ }^{\circ} \mathrm{C}$ under vacuum. After degassing, the solution was placed under $\mathrm{Ar}$ atmosphere, and a specific reaction temperature was set. A solution of hexamethyldisilathiane ((TMS $\left.)_{2} \mathrm{~S}\right)$ in ODE was quickly injected. After $6 \mathrm{~min}$, a second solution of (TMS) $)_{2} \mathrm{~S}$ in ODE was dropwise injected in a rate of $0.75 \mathrm{~mL} / \mathrm{min}$. After this second injection, heating was stopped, and the solution was let to naturally cool down to room temperature. QDs were purified three times by precipitation with acetone and ethanol and redispersed in anhydrous toluene. Finally, the concentration was adjusted to 30 
$\mathrm{mg} / \mathrm{mL}$, and the solution was bubbled with $\mathrm{N}_{2}$. Depending on the reaction parameters, $\mathrm{PbS}$ QDs of different sizes were synthesized.

n-Doped PbS QD Film Fabrication. PbS CQD films were deposited using a layer-by-layer spin-coating process under ambient conditions at $2500 \mathrm{rpm}$. For each layer, the CQD solution was deposited on semi-insulating $(10-20 \Omega \cdot \mathrm{cm})$ double-polished $\mathrm{Si}$. Solid-state ligand exchange was performed by flooding the surface with 1-ethyl-3-methylimidazolium iodide in methanol (EMII, $7 \mathrm{mg}$ / $\mathrm{mL}) 30 \mathrm{~s}$ before spin-coating dry at $2500 \mathrm{rpm}$. Two washes with methanol were used to remove unbound ligands. After film formation, samples are lightly $\mathrm{n}$-doped. ${ }^{16}$ Heavy $\mathrm{n}$-doping is achieved by infiltrating and capping the films with alumina $\left(\mathrm{Al}_{2} \mathrm{O}_{3}\right)$ via atomic layer deposition, following an already reported method. ${ }^{16}$

Measurement of the QD Doping Level. We employed a previously reported method to measure $n_{\mathrm{QD}}$ in our samples. ${ }^{16} n_{\mathrm{QD}}$ is extracted from the bleaching of the first exciton absorption peak upon heavy doping. Absorption was measured ambient conditions, using a Cary 5000 UV-vis-NIR spectrometer. If we define $I_{1}$ and $I_{2}$ as the integrated absorption strength of the first excitonic transition of the lightly doped and heavily doped samples, respectively, then $n_{\mathrm{QD}}=8(1$ $\left.-I_{2} / I_{1}\right)$. For related figures, see ref 16 .

Room-Temperature Absorption Measurements. Absorption of the thin films, $A_{\text {film }}$, was obtained (see eq 3) by means of reflection and transmission measurements, using a Cary 600 FTIR with microscope. The bench of the FTIR was purged with nitrogen gas to minimize the impact of atmospheric absorption in the measurements.

Calculation of the Absorption Coefficient, $\boldsymbol{\alpha}$. For the simple case of a thin slab of homogeneous material, $\alpha$ can be calculated by taking into consideration the multiple internal reflections that occur at the front and back air/sample interfaces, which increase the effective optical path. ${ }^{49}$ When the thin-film is deposited on a substrate, reflections taking place at every interface (refractive-index change), as well as absorption in the substrate, must be taken into account. For this case, $\alpha$ can also be analytically calculated assuming $\alpha t \ll 1,{ }^{50}$ where $t$ is the thickness of the thin film. However, in all cases the refractive index of the sample or, alternatively, the fraction of reflected light in a single air/sample interface must be known. Because of the wavelength range in our study $(5-9 \mu \mathrm{m})$, it was not possible for us to measure either of these values. Hence, we have calculated $\alpha$ using a simple model described by eq 8 :

$$
A_{\text {int }}=1-\mathrm{e}^{-\alpha t}
$$

where $A_{\text {int }}=A_{\text {film }} /\left(1-R_{\text {meas }}\right) . A_{\text {film }}$ is the measured absorption of the thin film, and $R_{\text {meas }}$ is the measured reflectance (see eq 3). $A_{\text {int }}$ represents the intrinsic absorption of the film-irrespective of the external medium - taking into account only the light that enters the film. To verify the validity of our model, we calculated $\alpha$ for samples of the same QDs but having different film thickness (Supporting Figure S11). The small difference in $\alpha$ among the different samples discards strong multiple-reflection effects in our measurements. The uncertainty in the determination of $\alpha$ can be explained in terms of errors propagated from the thickness and absorption measurements.

Temperature-Dependent Absorption Measurements. Absorption was obtained as 1 - transmission. Temperature variable transmission measurements were made under vacuum, using a Cary 610 FTIR with microscope coupled to a liquid-nitrogen cooled, temperature-controllable Linkam HFS350EV-PB4 stage equipped with $\mathrm{ZnSe}$ windows. For all samples, the temperature change was done at a controlled rate $(3 \mathrm{~K} / \mathrm{min})$. After reaching the temperature set point, temperature was stabilized $5 \mathrm{~min}$ prior to performing the measurements.

A bare-silicon substrate was used as a background sample. The same background, measured at RT, was used to determine the transmission of the samples at all temperatures. Low-temperature measurements exhibit a double-peak feature centered at $0.136 \mathrm{eV}$. This feature is due to the temperature-dependent energy shift of the $\mathrm{SiO}_{2}$ absorption in the samples, which is not corrected by the RT background.
Determination of Sample Thickness. The thicknesses of the samples used in absorption measurement were measured using a KLA-Tencor Alpha-Step IQ Surface Profiler.

\section{ASSOCIATED CONTENT}

\section{Supporting Information}

The Supporting Information is available free of charge at https://pubs.acs.org/doi/10.1021/acsnano.0c02033.

Example of thin-film absorption measurements, validation of the absorption model, bleach of exciton upon doping, fits of temperature dependence of interband and intraband transitions, absorption spectrum of silicon substrate and alumina, energy shift of the first exciton in doped QDs, relevant parameters of the intraband samples analyzed (PDF)

\section{AUTHOR INFORMATION}

\section{Corresponding Authors}

Iñigo Ramiro - Institut de Ciències Fotòniques (ICFO), The

Barcelona Institute of Science and Technology, 08860

Barcelona, Spain; 이이이.org/0000-0002-9663-4002;

Email: i.ramiro@ies.upm.es

Gerasimos Konstantatos - Institut de Ciencies Fotòniques (ICFO), The Barcelona Institute of Science and Technology, 08860 Barcelona, Spain; Institució Catalana de Recerca $i$ Estudis Avançats (ICREA), 08010 Barcelona, Spain; (1) orcid.org/0000-0001-7701-8127;

Email: Gerasimos.Konstantatos@icfo.eu

\section{Authors}

Biswajit Kundu - Institut de Ciencies Fotòniques (ICFO), The Barcelona Institute of Science and Technology, 08860 Barcelona, Spain

Mariona Dalmases - Institut de Ciencies Fotòniques (ICFO), The Barcelona Institute of Science and Technology, 08860 Barcelona, Spain

Onur Özdemir - Institut de Ciencies Fotòniques (ICFO), The Barcelona Institute of Science and Technology, 08860 Barcelona, Spain; (1) orcid.org/0000-0001-8692-5087

María Pedrosa - Institut de Ciencies Fotoniques (ICFO), The Barcelona Institute of Science and Technology, 08860 Barcelona, Spain

Complete contact information is available at: https://pubs.acs.org/10.1021/acsnano.0c02033

\section{Author Contributions}

I.R. designed the experiments and analyzed the results. I.R., B.K., and M.P. performed the absorption measurements. M.D. synthesized the QDs. I.R. and O.Ö. fabricated the samples. I.R. and G.K. wrote the manuscript with input from all authors. G.K. designed the experiments and supervised the work.

\section{Notes}

The authors declare no competing financial interest.

\section{ACKNOWLEDGMENTS}

We thank Dr. Iacopo Torre for helpful discussions. The authors acknowledge financial support from the European Research Council (ERC) under the European Union's Horizon 2020 research and innovation program (grant agreement no. 725165), the Spanish Ministry of Economy and Competitiveness (MINECO), and the "Fondo Europeo de Desarrollo Regional” (FEDER) through grant TEC2017- 
88655-R. The authors also acknowledge financial support from Fundacio Privada Cellex, the program CERCA and from the Spanish Ministry of Economy and Competitiveness, through the "Severo Ochoa" Program for Centres of Excellence.

\section{REFERENCES}

(1) Shirasaki, Y.; Supran, G. J.; Bawendi, M. G.; Bulović, V. Emergence of Colloidal Quantum-Dot Light-Emitting Technologies. Nat. Photonics 2013, 7, 13-23.

(2) Konstantatos, G.; Sargent, E. H. Colloidal Quantum Dot Photodetectors. Infrared Phys. Technol. 2011, 54, 278-282.

(3) Carey, G. H.; Abdelhady, A. L.; Ning, Z.; Thon, S. M.; Bakr, O. M.; Sargent, E. H. Colloidal Quantum Dot Solar Cells. Chem. Rev. 2015, 115, 12732-12763.

(4) Hetsch, F.; Zhao, N.; Kershaw, S. V.; Rogach, A. L. Quantum Dot Field Effect Transistors. Mater. Today 2013, 16, 312-325.

(5) Murray, C. B.; Norris, D. J.; Bawendi, M. G. Synthesis and Characterization of Nearly Monodisperse CdE $(E=S, S e, T e)$ Semiconductor Nanocrystallites. J. Am. Chem. Soc. 1993, 115, 87068715.

(6) Moreels, I.; Lambert, K.; Smeets, D.; De Muynck, D.; Nollet, T.; Martins, J. C.; Vanhaecke, F.; Vantomme, A.; Delerue, C.; Allan, G.; Hens, Z. Size-Dependent Optical Properties of Colloidal PbS Quantum Dots. ACS Nano 2009, 3, 3023-3030.

(7) Keuleyan, S. E.; Guyot-Sionnest, P.; Delerue, C.; Allan, G. Mercury Telluride Colloidal Quantum Dots: Electronic Structure, Size-Dependent Spectra, and Photocurrent Detection up to $12 \mathrm{Mm}$. ACS Nano 2014, 8, 8676-8682.

(8) Shim, M.; Guyot-Sionnest, P. N-Type Colloidal Semiconductor Nanocrystals. Nature 2000, 407, 981-983.

(9) Wang, C.; Shim, M.; Guyot-Sionnest, P. Electrochromic Nanocrystal Quantum Dots. Science 2001, 291, 2390-2392.

(10) Wehrenberg, B. L.; Wang, C.; Guyot-Sionnest, P. Interband and Intraband Optical Studies of PbSe Colloidal Quantum Dots. J. Phys. Chem. B 2002, 106, 10634-10640.

(11) Jagtap, A.; Livache, C.; Martinez, B.; Qu, J.; Chu, A.; Gréboval, C.; Goubet, N.; Lhuillier, E. Emergence of Intraband Transitions in Colloidal Nanocrystals [Invited]. Opt. Mater. Express 2018, 8, 1174.

(12) Rogalski, A. Recent Progress in Infrared Detector Technologies. Infrared Phys. Technol. 2011, 54, 136-154.

(13) Kim, J.; Choi, D.; Jeong, K. S. Self-Doped Colloidal Semiconductor Nanocrystals with Intraband Transitions in Steady State. Chem. Commun. 2018, 54, 8435-8445.

(14) Deng, Z.; Jeong, K. S.; Guyot-Sionnest, P. Colloidal Quantum Dots Intraband Photodetectors. ACS Nano 2014, 8, 11707-11714.

(15) Livache, C.; Martinez, B.; Goubet, N.; Gréboval, C.; Qu, J.; Chu, A.; Royer, S.; Ithurria, S.; Silly, M. G.; Dubertret, B.; Lhuillier, E. A Colloidal Quantum Dot Infrared Photodetector and Its Use for Intraband Detection. Nat. Commun. 2019, 10, 2125.

(16) Christodoulou, S.; Ramiro, I.; Othonos, A.; Figueroba, A.; Dalmases, M.; Özdemir, O.; Pradhan, S.; Itskos, G.; Konstantatos, G. Single-Exciton Gain and Stimulated Emission Across the Infrared Optical Telecom Band from Robust Heavily-Doped PbS Colloidal Quantum Dots. arXiv (Applied Physics), August 10, 2019, 1908.03796, ver. 1. https://arxiv.org/abs/1908.03796 (accessed 2020/01/20).

(17) Ramiro, I.; Özdemir, O.; Christodoulou, S.; Gupta, S.; Dalmases, M.; Torre, I.; Konstantatos, G. Mid- and Long-Wave Infrared Optoelectronics via Intraband Transitions in $\mathrm{PbS}$ Colloidal Quantum Dots. Nano Lett. 2020, 20, 1003-1008.

(18) Abelson, A.; Qian, C.; Salk, T.; Luan, Z.; Fu, K.; Zheng, J. G.; Wardini, J. L.; Law, M. Collective Topo-Epitaxy in the Self-Assembly of a 3D Quantum Dot Superlattice. Nat. Mater. 2020, 19, 49-55.

(19) Thimsen, E.; Johnson, M.; Zhang, X.; Wagner, A. J.; Mkhoyan, K. A.; Kortshagen, U. R.; Aydil, E. S. High Electron Mobility in Thin Films Formed via Supersonic Impact Deposition of Nanocrystals Synthesized in Nonthermal Plasmas. Nat. Commun. 2014, 5, 5822.
(20) Ephraim, J.; Lanigan, D.; Staller, C.; Milliron, D. J.; Thimsen, E. Transparent Conductive Oxide Nanocrystals Coated with Insulators by Atomic Layer Deposition. Chem. Mater. 2016, 28, 5549-5553.

(21) Cademartiri, L.; Montanari, E.; Calestani, G.; Migliori, A.; Guagliardi, A.; Ozin, G. A. Size-Dependent Extinction Coefficients of PbS Quantum Dots. J. Am. Chem. Soc. 2006, 128, 10337-10346.

(22) Diaconescu, B.; Padilha, L. A.; Nagpal, P.; Swartzentruber, B. S.; Klimov, V. I. Measurement of Electronic States of PbS Nanocrystal Quantum Dots Using Scanning Tunneling Spectroscopy: The Role of Parity Selection Rules in Optical Absorption. Phys. Rev. Lett. 2013, 110, 127406.

(23) Nootz, G.; Padilha, L. A.; Olszak, P. D.; Webster, S.; Hagan, D. J.; Van Stryland, E. W.; Levina, L.; Sukhovatkin, V.; Brzozowski, L.; Sargent, E. H. Role of Symmetry Breaking on the Optical Transitions in Lead-Salt Quantum Dots. Nano Lett. 2010, 10, 3577-3582.

(24) Kang, I.; Wise, F. W. Electronic Structure and Optical Properties of PbS and PbSe Quantum Dots. J. Opt. Soc. Am. B 1997, $14,1632$.

(25) Beygi, H.; Sajjadi, S. A.; Babakhani, A.; Young, J. F.; van Veggel, F. C. J. M. Surface Chemistry of As-Synthesized and Air-Oxidized PbS Quantum Dots. Appl. Surf. Sci. 2018, 457, 1-10.

(26) Gibbs, Z. M.; Kim, H.; Wang, H.; White, R. L.; Drymiotis, F.; Kaviany, M.; Jeffrey Snyder, G. Temperature Dependent Band Gap in $\mathrm{PbX}(\mathrm{X}=\mathrm{S}, \mathrm{Se}, \mathrm{Te})$. Appl. Phys. Lett. 2013, 103, 262109.

(27) Hens, Z.; Moreels, I. Light Absorption by Colloidal Semiconductor Quantum Dots. J. Mater. Chem. 2012, 22, 1040610415.

(28) Wang, Y.; Herron, N. Nanometer-Sized Semiconductor Clusters: Materials Synthesis, Quantum Size Effects, and Photophysical Properties. J. Phys. Chem. 1991, 95, 525-532.

(29) Rinehart, J. D.; Schimpf, A. M.; Weaver, A. L.; Cohn, A. W.; Gamelin, D. R. Photochemical Electronic Doping of Colloidal CdSe Nanocrystals. J. Am. Chem. Soc. 2013, 135, 18782-18785.

(30) De Sousa, J. S.; Leburton, J. P.; Freire, V. N.; Da Silva, E. F. Intraband Absorption and Stark Effect in Silicon Nanocrystals. Phys. Rev. B: Condens. Matter Mater. Phys. 2005, 72, 155438.

(31) Moreels, I.; Lambert, K.; De Muynck, D.; Vanhaecke, F.; Poelman, D.; Martins, J. C.; Allan, G.; Hens, Z. Composition and Size-Dependent Extinction Coefficient of Colloidal PbSe Quantum Dots. Chem. Mater. 2007, 19, 6101-6106.

(32) Leatherdale, C. A.; Woo, W. K.; Mikulec, F. V.; Bawendi, M. G. On the Absorption Cross Section of CdSe Nanocrystal Quantum Dots. J. Phys. Chem. B 2002, 106, 7619-7622.

(33) Talapin, D. V.; Gaponik, N.; Borchert, H.; Rogach, A. L.; Haase, M.; Weller, H. Etching of Colloidal InP Nanocrystals with Fluorides: Photochemical Nature of the Process Resulting in High Photoluminescence Efficiency. J. Phys. Chem. B 2002, 106, 1265912663.

(34) Yu, P.; Beard, M. C.; Ellingson, R. J.; Ferrere, S.; Curtis, C.; Drexler, J.; Luiszer, F.; Nozik, A. J. Absorption Cross-Section and Related Optical Properties of Colloidal InAs Quantum Dots. J. Phys. Chem. B 2005, 109, 7084-7087.

(35) Karel Capek, R.; Moreels, I.; Lambert, K.; De Muynck, D.; Zhao, Q.; Van Tomme, A.; Vanhaecke, F.; Hens, Z. Optical Properties of Zincblende Cadmium Selenide Quantum Dots. J. Phys. Chem. C 2010, 114, 6371-6376.

(36) Kamal, J. S.; Omari, A.; Van Hoecke, K.; Zhao, Q.; Vantomme, A.; Vanhaecke, F.; Capek, R. K.; Hens, Z. Size-Dependent Optical Properties of Zinc Blende Cadmium Telluride Quantum Dots. J. Phys. Chem. C 2012, 116, 5049-5054.

(37) Vossmeyer, T.; Katsikas, L.; Giersig, M.; Popovic, I. G.; Diesner, K.; Chemseddine, A.; Eychmüller, A.; Weller, H. CdS Nanoclusters: Synthesis, Characterization, Size Dependent Oscillator Strength, Temperature Shift of the Excitonic Transition Energy, and Reversible Absorbance Shift. J. Phys. Chem. 1994, 98, 7665-7673.

(38) Şahin, M. Photoionization Cross Section and Intersublevel Transitions in a One- and Two-Electron Spherical Quantum Dot with a Hydrogenic Impurity. Phys. Rev. B: Condens. Matter Mater. Phys. 2008, 77, 045317. 
(39) Yilmaz, S.; Şafak, H. Oscillator Strengths for the Intersubband Transitions in a CdS-SiO2 Quantum Dot with Hydrogenic Impurity. Phys. E 2007, 36, 40-44.

(40) Olkhovets, A.; Hsu, R. C.; Lipovskii, A.; Wise, F. W. SizeDependent Temperature Variation of the Energy Gap in Lead-Salt Quantum Dots. Phys. Rev. Lett. 1998, 81, 3539-3542.

(41) Dey, P.; Paul, J.; Bylsma, J.; Karaiskaj, D.; Luther, J.M.; Beard, M.C.; Romero, A.H. Origin of the Temperature Dependence of the Band Gap of $\mathrm{PbS}$ and $\mathrm{PbSe}$ Quantum Dots. Solid State Commun. 2013, 165, 49-54.

(42) Nordin, M. N.; Li, J.; Clowes, S. K.; Curry, R. J. Temperature Dependent Optical Properties of PbS Nanocrystals. Nanotechnology 2012, 23, 275701.

(43) Ullrich, B.; Wang, J. S.; Brown, G. J. Analysis of Thermal Band Gap Variations of PbS Quantum Dots by Fourier Transform Transmission and Emission Spectroscopy. Appl. Phys. Lett. 2011, 99, 081901.

(44) Liptay, T. J.; Ram, R. J. Temperature Dependence of the Exciton Transition in Semiconductor Quantum Dots. Appl. Phys. Lett. 2006, 89, 223132.

(45) Dai, Q.; Zhang, Y.; Wang, Y.; Hu, M. Z.; Zou, B.; Wang, Y.; Yu, W. W. Size-Dependent Temperature Effects on PbSe Nanocrystals. Langmuir 2010, 26, 11435-11440.

(46) Luque, A.; Martí, A.; Stanley, C. Understanding IntermediateBand Solar Cells. Nat. Photonics 2012, 6, 146-152.

(47) Ramiro, I.; Marti, A.; Antolin, E.; Luque, A. Review of Experimental Results Related to the Operation of Intermediate Band Solar Cells. IEEE J. Photovolt. 2014, 4, 736-748.

(48) Lee, J. W.; Kim, D. Y.; Baek, S.; Yu, H.; So, F. Inorganic UVVisible-SWIR Broadband Photodetector Based on Monodisperse PbS Nanocrystals. Small 2016, 12, 1328-1333.

(49) Pankove, J. I. Optical Processes in Semicondcutors; Dover Publications, Inc: New York, 1975; pp 93-94.

(50) Bubenzer, A.; Koidl, P. Exact Expressions for Calculating ThinFilm Absorption Coefficients from Laser Calorimetric Data. Appl. Opt. 1984, 23, 2886. 